PROCEEDINGS OF THE

AMERICAN MATHEMATICAL SOCIETY

Volume 134, Number 4, Pages 1059-1063

S 0002-9939(05)08076-7

Article electronically published on July 20, 2005

\title{
DYNAMICAL SYSTEMS METHOD (DSM) FOR UNBOUNDED OPERATORS
}

\author{
A. G. RAMM
}

(Communicated by Joseph A. Ball)

\begin{abstract}
Let $L$ be an unbounded linear operator in a real Hilbert space $H$, a generator of a $C_{0}$ semigroup, and let $g: H \rightarrow H$ be a $C_{l o c}^{2}$ nonlinear map. The DSM (dynamical systems method) for solving equation $F(v):=L v+g(v)=0$ consists of solving the Cauchy problem $\dot{u}=\Phi(t, u), u(0)=u_{0}$, where $\Phi$ is a suitable operator, and proving that i) $\exists u(t) \forall t>0$, ii) $\exists u(\infty)$, and iii) $F(u(\infty))=0$.

Conditions on $L$ and $g$ are given which allow one to choose $\Phi$ such that i), ii), and iii) hold.
\end{abstract}

\section{INTRODUCTION}

Let $H$ be a real Hilbert space, let $L$ be a linear, densely defined in $H$, closed operator, a generator of a $C_{0}$ semigroup (see [2]), and let $g: H \rightarrow H$ be a nonlinear $C_{l o c}^{2}$ map, i.e.,

$$
\sup _{u \in B\left(u_{0}, R\right)}\left\|g^{(j)}(u)\right\| \leq M_{j}(R), \quad j=0,1,2, \quad B\left(u_{0}, R\right):=\left\{u:\left\|u-u_{0}\right\| \leq R\right\},
$$

where $g^{(j)}$ is the Fréchet derivative of order $j, R>0$ is some number, $M_{j}(R)$ are some positive constants, and $u_{0} \in H$ is some element. In many applications the problems can be formulated as the following operator equation:

$$
F(v):=L v+g(v)=0 .
$$

We want to study this equation by the dynamical systems method (DSM), which also allows one to develop numerical methods for solving equation (1.2). The DSM for solving equation (1.2) consists of solving the problem

$$
\dot{u}=\Phi(t, u), \quad u(0)=u_{0},
$$

where $\dot{u}:=\frac{d u}{d t}$, and $\Phi(t, u)$ is a nonlinear operator chosen so that problem (1.3) has a unique global solution which stabilizes at infinity to the solution of equation (1.2):
i) $\exists u(t) \forall t>0$,
ii) $\exists u(\infty)$,
iii) $F(u(\infty))=0$.

Received by the editors February 18, 2004 and, in revised form, October 26, 2004.

2000 Mathematics Subject Classification. Primary 35R25, 35R30, 37B55, 47H20, 47J05, 49N45, 65M32, 65R30.

Key words and phrases. Dynamical systems method, nonlinear operator equations, ill-posed problems.

(C)2005 American Mathematical Society 
In 2 the DSM has been studied and justified for $F \in C_{l o c}^{2}$ and

$$
\sup _{u \in B\left(u_{0}, R\right)}\left\|\left[F^{\prime}(u)\right]^{-1}\right\| \leq m_{1}
$$

for monotone $F \in C_{l o c}^{2}$; for monotone hemicontinuous defined on all of $H$ operators $F$; for non-monotone $F \in C_{l o c}^{2}$ such that there exists a $y$ such that $F(y)=0$ and the operator $A:=F^{\prime}(y)$ maps any ball $B(0, r)$ centered at the origin and of sufficiently small radius $r>0$ into a set which has a nonempty intersection with $B(0, R)$; and for $F \in C_{l o c}^{2}$ satisfying a spectral condition: $\left\|\left(F^{\prime}(u)+\varepsilon\right)^{-1}\right\| \leq(c \varepsilon)^{-1}, 0<c \leq 1$, in which case $F$ is replaced by $F+\varepsilon I$ in (1.3), and then $\varepsilon$ is taken to zero.

In this paper the DSM is justified for a class of nonlinear unbounded operators of the type $L+g$, where $L$ is a generator of a $C_{0}$ semigroup, $g \in C_{l o c}^{2}$, and some suitable additional assumptions are made.

Which assumptions are suitable? A simple example is

$$
\left\|L^{-1}\right\| \leq m \text {. }
$$

If (1.6) holds, then (1.2) is equivalent to

$$
f(u):=u+L^{-1} g(u)=0, \quad f \in C_{l o c}^{2} .
$$

Assume

$$
\sup _{u \in B\left(u_{0}, R\right)}\left\|\left[I+L^{-1} g^{\prime}(u)\right]^{-1}\right\| \leq m_{1} .
$$

This assumption, holds, e.g., if $L^{-1} g^{\prime}(u)$ is a compact operator in $H$ for any $u \in$ $B\left(u_{0}, R\right)$ and the operator $I+L^{-1} g^{\prime}(u)$ is injective.

Our first result is the following theorem.

Theorem 1.1. Assume (1.6), (1.8), and let $\Phi:=-\left[I+L^{-1} g^{\prime}(u)\right]^{-1}\left[u+L^{-1} g(u)\right]$. If

$$
\left\|u_{0}+L^{-1} g\left(u_{0}\right)\right\| m_{1} \leq R
$$

then equation (1.2) has a unique solution $v \in B\left(u_{0}, R\right)$, the conclusions $\left.\mathrm{i}\right)$, ii), and iii) hold for (1.3), and $u(\infty)=v$.

Remark 1.1. If $L$ is not boundedly invertible, i.e., (1.6) fails, then one can use the following assumption (A).

Assumption A. There exists a sector $S=\{z: 0<|z| \leq a,|\arg z-\pi| \leq \delta\}$, which consists of regular points of $L$. Here $a>0$ and $\delta>0$ are arbitrary small fixed numbers.

If Assumption (A) holds, then

$$
\left\|(L+\varepsilon)^{-1}\right\| \leq \frac{1}{\varepsilon \sin (\delta)} .
$$

This estimate holds in particular if $L=L^{*} \geq 0$, and in this case $\sin (\delta)=1$.

The following theorem is our next result:

Theorem 1.2. Assume that $L^{*}=L \geq 0$ is a densely defined linear operator, $g \in C_{l o c}^{2}, g^{\prime}(u) \geq 0 \forall u \in H$, equation (1.2) is solvable and $v$ is its (unique) minimal-norm solution. Define $\Phi(u)=-\left[I+(L+\varepsilon)^{-1} g^{\prime}(u)\right]^{-1}\left[u+(L+\varepsilon)^{-1} g(u)\right]$, $\varepsilon=$ const $>0$. Assume that (1.8) holds with $L_{\varepsilon}:=L+\varepsilon I$ replacing $L$, and $m_{1}=m_{1}(\varepsilon)>0$. Then problem (1.3) has a unique global solution $u_{\varepsilon}(t)$, there 
exists $u_{\varepsilon}(\infty):=v_{\varepsilon}$, and $F\left(v_{\varepsilon}\right)+\varepsilon v_{\varepsilon}:=L v_{\varepsilon}+g\left(v_{\varepsilon}\right)+\varepsilon v_{\varepsilon}=0$. Moreover, there exists the limit $\lim _{\varepsilon \rightarrow 0} v_{\varepsilon}=v$, which is the unique minimal-norm solution to (1.2).

In Section 2 proofs are given.

\section{Proofs}

Proof of Theorem 1.1. If $\Phi=-\left[I+L^{-1} g^{\prime}(u)\right]^{-1}\left[u+L^{-1} g(u)\right]$ and

$$
p(t):=\left\|u+L^{-1} g(u)\right\|,
$$

then $p \dot{p}=-p^{2}$. Thus

$$
p(t)=p(0) e^{-t}
$$

From (2.1), (1.8) and (1.3) one gets

$$
\|\dot{u}\| \leq m_{1} p(0) e^{-t}, \quad p(0)=\left\|u_{0}+L^{-1} g\left(u_{0}\right)\right\| .
$$

Inequality (2.2) implies the global existence of $u(t)$, the existence of $u(\infty):=$ $\lim _{t \rightarrow \infty} u(t)$, and the estimates

$$
\|u(t)-u(\infty)\| \leq m_{1} p(0) e^{-t}, \quad\left\|u(t)-u_{0}\right\| \leq m_{1} p(0) .
$$

If (1.9) holds, then (2.3) implies $\left\|u(t)-u_{0}\right\| \leq R$, so the trajectory $u(t)$ stays in the ball $B\left(u_{0}, R\right)$, that is, $u(t) \in B\left(u_{0}, R\right) \forall t \geq 0$. Passing to the limit $t \rightarrow \infty$ in equation (1.3) yields

$$
0=-\left[I+L^{-1} g^{\prime}(u(\infty))\right]^{-1}\left[u(\infty)+L^{-1} g(u(\infty))\right] .
$$

Thus $u(\infty):=v$ solves the equation $v+L^{-1} g(v)=0$, so $v$ solves (1.2), and therefore i), ii) and iii) hold. Theorem 1.1 is proved.

Proof of Theorem 1.2. If $L^{*}=L \geq 0$ in $H$ and $g^{\prime}(u) \geq 0$, then, for any $\varepsilon>0$, Theorem 1.1 yields the existence of a unique solution $v_{\varepsilon}$ to equation (1.2) with $L$ replaced by $L+\varepsilon I$. This solution $v_{\varepsilon}=u_{\varepsilon}(\infty)$, where $u_{\varepsilon}(t)$ is the solution to (1.3) with

$$
\Phi=-\left[I+(L+\varepsilon)^{-1} g^{\prime}(u)\right]^{-1}\left[u+(L+\varepsilon)^{-1} g(u)\right] .
$$

Let us prove that $\lim _{\varepsilon \rightarrow 0} v_{\varepsilon}=v$, where $v$ solves (1.2). We do not assume that (1.2) has a unique solution.

Let $v_{\varepsilon}-v:=w$. Then $L w+\varepsilon v_{\varepsilon}+g\left(v_{\varepsilon}\right)-g(v)=0$, so, using the assumptions $L \geq 0$ and $g^{\prime}(u) \geq 0$, one gets $\varepsilon\left(v_{\varepsilon}, v_{\varepsilon}-v\right) \leq 0,\left\|v_{\varepsilon}\right\|^{2} \leq\left\|v_{\varepsilon}\right\|\|v\|$, and $\left\|v_{\varepsilon}\right\| \leq\|v\|, \forall \varepsilon>0$. Thus $v_{\varepsilon} \rightarrow v_{0}$ and $L v_{\varepsilon}+g\left(v_{\varepsilon}\right) \rightarrow 0$, where $\rightarrow$ stands for the weak convergence in $H$ and the convergent subsequence is again denoted $v_{\varepsilon}$.

In the above argument the element $v$ can be an arbitrary element in the set $N_{F}:=\{v: L v+g(v)=0\}$. Thus, we have proved that $\left\|v_{0}\right\| \leq\|v\|$ for all $v \in N_{F}$.

Let us prove that $L\left(v_{0}\right)+g\left(v_{0}\right)=0$, i.e., $v_{0} \in N_{F}$.

Assume first that $v_{0} \in D(L)$. We prove this assumption later. The monotonicity of $L+g$ yields

$$
\left(L\left(v_{\varepsilon}\right)+g\left(v_{\varepsilon}\right)+\varepsilon v_{\varepsilon}-L\left(v_{0}-t z\right)-g\left(v_{0}-t z\right)-\varepsilon\left(v_{0}-t z\right), v_{\varepsilon}-v_{0}+t z\right) \geq 0
$$

where $t>0$, and $z \in D(L)$ is arbitrary. Let $\varepsilon \rightarrow 0$ in (2.5). Then, using $v_{\varepsilon} \rightarrow v_{0}$ and $L v_{\varepsilon}+g\left(v_{\varepsilon}\right) \rightarrow 0$, one gets

$$
\left(-L\left(v_{0}-t z\right)-g\left(v_{0}-t z\right), \quad z\right) \geq 0 \quad \forall z \in D(L) .
$$

Let $t \rightarrow 0$ in (2.6). Then $\left(L v_{0}+g\left(v_{0}\right), z\right) \leq 0 \quad \forall z \in D(L)$. Since $D(L)$ is dense in $H$, it follows that $L v_{0}+g\left(v_{0}\right)=0$, so $v_{0} \in N_{F}$. 
We have proved above that $\left\|v_{0}\right\| \leq\|v\|$ for any $v \in N_{F}$. Because $N_{F}$ is a closed and convex set, as we prove below, and $H$ is a uniformly convex space, there is a unique element $v \in N_{F}$ with minimal norm. Therefore, it follows that $v_{0}=v$, where $v$ is the minimal-norm solution to (1.2) and $v_{0}$ is the weak limit of $v_{\varepsilon}$.

Let us prove the strong convergence $v_{\varepsilon} \rightarrow v$.

We know that $v_{\varepsilon} \rightarrow v$. The inequality $\left\|v_{\varepsilon}\right\| \leq\|v\|$ implies

$$
\|v\| \leq \lim \inf _{\varepsilon \rightarrow 0}\left\|v_{\varepsilon}\right\| \leq \lim \sup _{\varepsilon \rightarrow 0}\left\|v_{\varepsilon}\right\| \leq\|v\| .
$$

Therefore $\lim _{\varepsilon \rightarrow 0}\left\|v_{\varepsilon}\right\|=\|v\|$. Consequently one gets

$$
\lim _{\varepsilon \rightarrow 0}\left\|v_{\varepsilon}-v\right\|^{2}=\lim _{\varepsilon \rightarrow 0}\left[\left\|v_{\varepsilon}\right\|^{2}+\|v\|^{2}-2 \Re\left(v_{\varepsilon}, v\right)\right] \leq 2\left[\|v\|^{2}-(v, v)\right]=0 .
$$

Thus, $v_{\varepsilon} \rightarrow v$, as claimed.

Since $g$ is continuous, it follows that $g\left(v_{\varepsilon}\right) \rightarrow g(v)$.

The equation

$$
L v_{\varepsilon}+\varepsilon v_{\varepsilon}+g\left(v_{\varepsilon}\right)=0,
$$

the inequality $\left\|v_{\varepsilon}\right\| \leq\|v\|$, and the relation $g\left(v_{\varepsilon}\right) \rightarrow g(v)$ imply $L v_{\varepsilon} \rightarrow \eta:=-g(v)$.

Let us prove that $v_{0} \in D(L)$.

Because $v_{0}=v$, it is sufficient to check that $v \in D(L)$. Since $L$ is selfadjoint and $L v_{\varepsilon} \rightarrow \eta$, one has

$$
(\eta, \psi)=\lim _{\varepsilon \rightarrow 0}\left(L v_{\varepsilon}, \psi\right)=\lim _{\varepsilon \rightarrow 0}\left(v_{\varepsilon}, L \psi\right)=(v, L \psi) \quad \forall \psi \in D(L) .
$$

Thus $v \in D(L)$ and $L v=\eta$.

Let us finally check that $N_{F}$ is closed and convex.

Assume $F\left(v_{n}\right):=L v_{n}+g\left(v_{n}\right)=0, v_{n} \rightarrow v$. Then $g\left(v_{n}\right) \rightarrow g(v)$ because $g$ is continuous. Thus, $L v_{n} \rightarrow \eta:=-g(v)$. Since $L$ is closed, the relations $v_{n} \rightarrow v$ and $L v_{n} \rightarrow \eta$ imply $L v=\eta=-g(v)$. So, $v \in N_{F}$, and consequently $N_{F}$ is closed.

Assume that $0<s<1, v, w \in N_{F}$ and $\psi=s v+(1-s) w$. Let us show that $\psi \in N_{F}$. One has $w \in N_{F}$ if and only if

$$
(F(z), z-w) \geq 0 \quad \forall z \in D(L) .
$$

Indeed, if $F(w)=0$, then $F(z)=F(z)-F(w)$, and the above equation holds because $F$ is a monotone operator. Conversely, if this equation holds, then take $z-w=t \eta, t=$ const $>0, \eta \in D(L)$ is arbitrary, and get $(F(w+t \eta), \eta) \geq 0$. Let $t \rightarrow 0$; then $(F(w), \eta) \geq 0 \forall \eta \in D(L)$. Thus, since $\overline{D(L)}=H$, one gets $w \in N_{F}$. If $\psi=s v+(1-s) w$, and $v, w \in N(F)$, then $(F(z), z-s v-(1-s) w)=$ $s(F(z), z-v)+(1-s)(F(z), z-w) \geq 0$. Thus, $N_{F}$ is convex.

Theorem 1.2 is proved.

Remark 2.1. Theorem 1.2 gives a theoretical framework for a study of nonlinear ill-posed operator equations $F(u)=f$, with $F(u)=L u+g(u)$, where the operator $F^{\prime}(u)$ is not boundedly invertible. In this short paper, we do not discuss the case when the data $f$ are given with some error: $f_{\delta}$ is given in place of $f,\left\|f_{\delta}-f\right\| \leq \delta$. In this case one can use DSM for a stable solution of the equation $F(v)=f$ with noisy data $f_{\delta}$, and one uses an algorithm for choosing stopping time $t_{\delta}$ such that $\lim _{\delta \rightarrow 0}\left\|u_{\delta}\left(t_{\delta}\right)-v\right\|=0$, where $u_{\delta}(t)$ is the solution to (1.3) with $\Phi=\Phi_{\delta}$ chosen suitably (see [3]). 
Remark 2.2. Under the assumptions of Theorem 1.1 one of the many possibilities to choose $\Phi$ in (1.3) so that the DSM can be justified, that is, (1.4) is valid, is the the choice made in this theorem, the choice which can be interpreted as a continuous analog of the Newton's method. The classical Newton's method for solving an equation $G(u)=0$ consists in calculating the sequence $u_{n+1}=u_{n}-\left[G^{\prime}\left(u_{n}\right)\right]^{-1} G\left(u_{n}\right), \quad u_{0}=u_{0}$, where the initial approximation $u_{0}$ is given, and one assumes that $\left\|\left[G^{\prime}(u)\right]^{-1}\right\| \leq m_{1}$, which is an analog of condition (1.5). Under certain assumptions (see, e.g., [1]), one can prove that the limit $\lim _{n \rightarrow \infty} u_{n}=u$ solves the equation $G(u)=0$. If one takes $G(u):=u+L^{-1} g(u)$, then $G^{\prime}(u) w=\left[I+L^{-1} g^{\prime}(u)\right] w$, and problem (1.3) with $\Phi=-\left[G^{\prime}(u)\right]^{-1} G(u)$ is a continuous analog of the Newton's method. The assumption (1.9) can be written as $\left\|G\left(u_{0}\right)\right\| m_{1} \leq R$. By Theorem 1.1, this assumption together with the assumption $\sup _{u \in B\left(u_{0}, R\right)}\left\|G^{(j)}(u)\right\| \leq M_{j}, \quad j=0,1,2, \quad B\left(u_{0}, R\right):=\left\{u:\left\|u-u_{0}\right\| \leq R\right\}$, imply the existence of the unique solution to problem (1.3) with $\Phi=-\left[G^{\prime}(u)\right]^{-1} G(u)$ and the validity of conclusions (1.4) for this solution. This means that the DSM is justified for the solution to the equation $G(u)=0$ under the above assumptions, and the DSM can be viewed for this particular choice of $\Phi$ as a continuous analog of Newton's method. The basic condition $\left\|G\left(u_{0}\right)\right\| m_{1} \leq R$ for the existence of a solution to equation $G(u)=0$ is very simple, simpler than the classical conditions for the convergence of the discrete Newton's method (cf., e.g., [1]). Some numerical aspects of using DSM are discussed in [4. Theoretical aspects of the DSM are discussed in [3] and [5].

\section{REFERENCES}

[1] Deimling, K., Nonlinear functional analysis, Springer Verlag, Berlin, 1985. MR0787404 (86j:47001)

[2] Pazy, A., Semigroups of linear operators and applications to partial differential equations, Springer-Verlag, New York, 1983. MR0710486 (85g:47061)

[3] Ramm, A. G., Dynamical systems method for solving operator equations, Communic. in Nonlinear Sci. and Numer. Simulation, 9, N2, (2004), 383-402. MR2045643 (2004m:47161)

[4] Ramm, A. G., Discrepancy principle for the dynamical systems method, Communic. in Nonlinear Sci. and Numer. Simulation, 10, N1, (2005), 95-101. MR2090273

[5] Ramm, A. G., Dynamical systems method (DSM) and nonlinear problems, in "Spectral Theory and Nonlinear Analysis" (editor J. Lopez-Gomez), World Scientific Publishers, Singapore, 2005.

Department of Mathematics, Kansas State University, Manhattan, Kansas 665062602

E-mail address: ramm@math.ksu.edu

URL: http://www.math.ksu.edu/ ramm 